Original Research Paper

\title{
Star Catalog Generation for Satellite Attitude Navigation Using Density Based Clustering
}

\author{
${ }^{1,3}$ Muhammad Arif Saifudin, ${ }^{2}$ Bib Paruhum Silalahi and ${ }^{1}$ Imas Sukaesih Sitanggang \\ ${ }^{1}$ Department of Computer Science, Bogor Agriculture University, Bogor, Indonesia \\ ${ }^{2}$ Department of Mathematics, Bogor Agriculture University, Bogor, Indonesia \\ ${ }^{3}$ Satellite Technology Center, Indonesian National Institute of Aeronautics and Space, Bogor, Indonesia
}

Article history

Received: 29-06-2015

Revised: 10-09-2015

Accepted: 26-01-2016

\section{Corresponding Author:}

Muhammad Arif Saifudin

Satellite Technology Center,

Indonesian National Institute of

Aeronautics and Space, Bogor,

Indonesia

Email: arif.saifudin@lapan.go.id

\begin{abstract}
A new method to generate star catalog using density-based clustering is proposed. It identifies regions of a high star density by using Density-Based Spatial Clustering of Application with Noise (DBSCAN) algorithm. Reducing the number stars performed by storing the brightest star in each cluster. The brightest star and all non-clustered members are then stored as a navigation star candidate. Monte Carlo simulation has performed to generate random FOV to check the uniformity of the new catalog. Succeed parameter is if there are at least three stars in the FOV. The simulation results compare between DBSCAN method and Magnitude Filtering Method (MFM) which is the common method to generate star catalog. The result shows that DBSCAN method is better than MFM such for number of star 846 DBSCAN has success $100 \%$ while MFM $95 \%$. It concluded that density-based clustering is a promising method to select navigation star for star catalog generation.
\end{abstract}

Keywords: Star Sensor, Star Catalog, Star Identification, Clustering Method, DBSCAN

\section{Introduction}

Star sensor is widely used as an attitude determination sensor in satellite since the star sensor is a most accurate satellite attitude determination sensor (Bak, 1999; Mohammadnejad et al., 2012). Star sensor provides 3-axis satellite orientation estimation in space. The advancement of star sensor technology makes it possible to consider practical implementation of satellite attitude determination based on the star sensor (Gai et al., 1985). Star sensor basically consists of an optical system (lens, detection sensor and baffle) and an Electronic Data Processing Unit (EDPU). The EDPU is a unit to process stars image received from the optical system and then calculate the position of the star (also known as star identification) in the celestial coordinate system. Star identification requires a star database or a star catalog to acquire the star position of the camera image. The position of stars then transformed as the attitude of the satellite. Star identification process for star pattern recognition needs a reference of stars or navigation stars as known as star catalog. If the pattern contained on or an identical sensor matches in the star catalog, the recognition process is successful. Otherwise, if the star identification process does not find any stars matched in the catalog, the star sensor failed in identifying stars. Therefore, the satellite did not acquire attitude at the times. Hence, in order to provide attitude information to satellite accurately and continuously, navigation stars must has number of navigation stars appearing in FOV must be enough to perform star identification and the total number of navigational stars is minimized. Moreover, the distribution of navigation stars should nearly uniform (Zhang et al., 2004). A common problem of star identification is to generate navigation star for star catalog. The number of stars of the catalog may affect to star identification process and accuracy of attitude determination. Less number of stars might speed up of the identification process, however the accuracy is low. The simplest method to generate star catalog is used Magnitude Filtering Method (MFM) (Kim and Junkins, 2002). In MFM, stars in base catalog have certain magnitude are selected as navigation stars. Generally, navigational stars generated by MFM are considering the sensitivity threshold of the sensor to the star magnitude. For instance, in the SAO J2000 star catalog records more than 250,000 stars information with the brightness value or star magnitude $\left(\mathrm{M}_{\mathrm{v}}\right)$ ranging from $-1.6 \mathrm{M}_{\mathrm{v}}$ as the brightest star to $10.0 \mathrm{M}_{\mathrm{v}}$ as the dimmest star. If the catalog extracted up to star magnitude $6.0 \mathrm{M}_{\mathrm{v}}$, the 
catalog contains 5103 stars and if the catalog extracted up to magnitude $5.0 \mathrm{M}_{\mathrm{v}}$, the catalog contains 1571 stars. This method has easy to apply, but this method is constrained in the uneven distribution of stars that allow the emergence of a "hole" in any FOV bore sight direction. In these conditions, the star sensor failed in identifying stars and affecting the attitude determination of the satellite. In fact, stars distribution on the celestial sphere is not nearly uniform. For instance, high density stars are located in the Milky Way region while few stars located in North Galactic Pole (Samaan et al., 2003). In order to reduce the number of stars for star catalog generation purpose, it requires a method to identify regions of a high star density then reduce a number of them. This process is repeated to obtain the number of stars in which the simulation in any FOV direction always gets at least three stars as a condition star pattern recognition by using triangle algorithm. The remaining stars are a navigation star candidate. The related work for star catalog generation has been introduced. Thinning Method (TM) (Kim and Junkins, 2002) was introduced to select navigation star candidates. However, TM is complicated to select adjacent navigation star candidate by using large argument as a criteria. Also, TM did not consider the visual magnitude threshold that can be detected by star sensor.

In this article, the new method to generate a star catalog of star sensor application using density-based clustering algorithm is introduced. The clustering algorithm that used is Density-Based Spatial Clustering of Application with Noise (DBSCAN). The basic idea is generating the new star catalog by reducing the number of stars from the base catalog. The reduction technique that applied is removing unnecessity stars located in the high density region and storing the stars located in the low density region. DBSCAN is one of partitional type clustering algorithm that could grouping objects with specified distance and minimum number of objects. In each cluster carried out a reduced number of stars. This algorithm is simple as it only requires two parameters those are the minimum number of stars in a cluster and radius of the cluster's core. In this study, a wide FOV $23^{\circ}$ circular is implemented in the simulation to evaluate the uniformity of the new catalog. Monte Carlo simulation used for both MFM and Clustering Method and then compare the number of simulations succeeds between those methods. The simulation has performed to generate random FOV in any bore sight direction and find at least three stars matched in the catalog.

\section{Methodology}

Star sensor works by identifying the stars that captured by camera then provide star position as attitude of satellite. The basic principle of star sensor is illustrated in Fig. 1. Various star identification

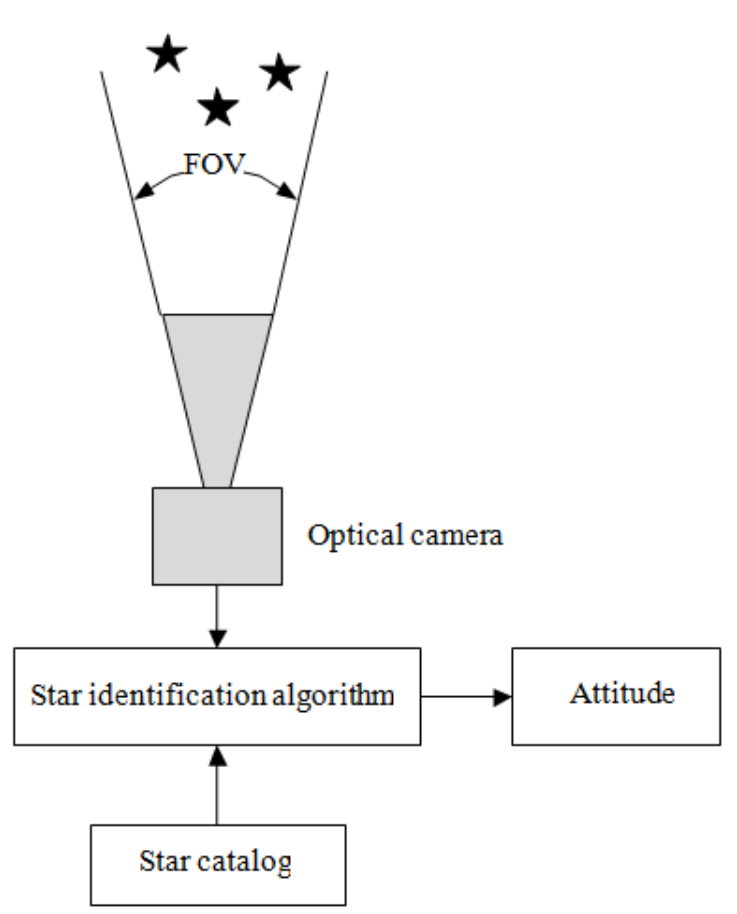

Fig. 1. Basic principle of star sensor

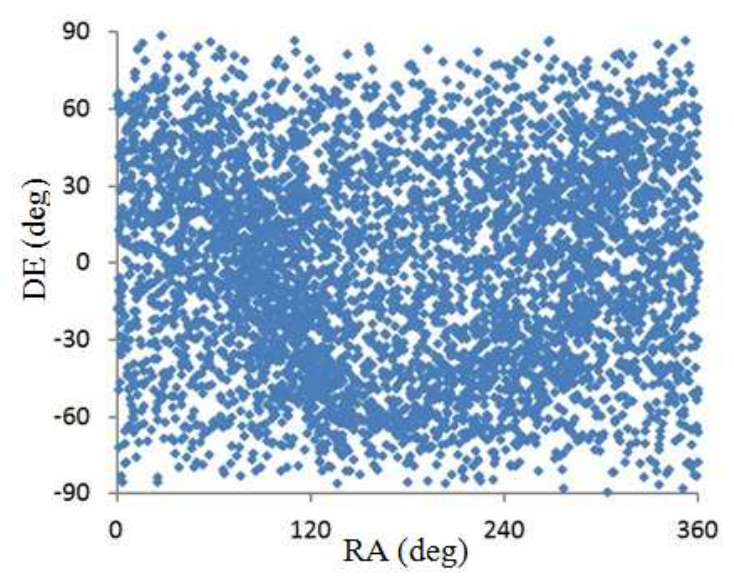

Fig. 2. Star catalog SAO J2000 with magnitude $6.0 \mathrm{M}_{\mathrm{v}}$

algorithms were proposed and they vary in complexity, recognition time, database or catalog size, recognition accuracy and robustness (Pham et al., 2013). The most algorithms have used a geometric approach of star cluster inside FOV. Common method is measuring the angular separation of each star to others. Such algorithms that used this method are Oriented Triangle (Rousseau et al., 2005), Planar Triangle (Cole and Crassidis, 2006) and Geometric Voting (Kolomenkin et al., 2008). Also, the new approach was introduced based on artificial intelligence, such Neural Network (Lindsey et al., 1997), Genetic Algorithm (Paladugu et al., 2003) and Fuzzy (Sohrabi and Shirazi, 2010). 


\begin{tabular}{lcc}
\multicolumn{4}{l}{ Table 1. Initial catalog format } \\
\hline Star ID & RA $(\mathrm{deg})$ & DE (deg) \\
\hline 1 & 101.287 & -16.716 \\
2 & 95.988 & -52.696 \\
3 & 279.235 & 38.784 \\
4 & 219.901 & -60.835 \\
5 & 79.172 & 45.998 \\
$\ldots$ & $\ldots$ & $\ldots$ \\
5102 & 354.600 & -76.870 \\
5103 & 356.106 & -70.490 \\
\hline
\end{tabular}

The original catalog used in proposed work is SAO J2000 that records more than 250,000 stars. In order to reduce the number of stars, then stars with magnitude 6.0 $\mathrm{M}_{\mathrm{v}}$ were selected as initial catalog since most sensors have a sensitivity of starlight with magnitude $6.0 \mathrm{M}_{\mathrm{v}}$. The first selection resulted 5103 stars as initial catalog and it plotted in 2D as shown is Fig. 2. The initial catalog used as the first dataset in clustering algorithm.

The information required in star catalog generation is star ID and star position information that is Right Ascension (RA) and Declination (DE). The definition of this star information will depict in the next section of this article. Format of Initial catalog is shown in Table 1. As the star clusters created, then making a selection for the stars in each cluster as a navigation star candidate. The new catalog, which has fewer numbers of stars, is generated. The resulting of new catalog from clustering method is tested for the uniformity using Monte Carlo simulation.

\section{DBSCAN Algorithm}

Clustering algorithm that used in this research is Density-Based Spatial Clustering of Application with Noise (DBSCAN) (Ester et al., 1996). DBSCAN is partitional type of clustering where areas with high density are considered as cluster and areas with low density are called noise (Nagpal and Mann, 2011). The key parameter of DBSCAN is a cluster has at least the minimum number of objects (MinPts) within a neighborhood radius (Eps). Criteria of cluster in DBSCAN are (Ester et al., 1996):

Core, core is the point as the center of the cluster, which has a number of neighboring objects more or equal to the value specified MinPts in the range Eps.

Border, border is the point of a number MinPts fewer yet has the adjacency relationship with the core point (core).

Noise, noise is neither both a core point and border point. Directly Density-Reachable, A point $\mathrm{p}$ is directly density-reachable from a point q with respect to Eps and MinPts if $p \in N_{E p s}(q)$ and $\left|N_{E p s}(q)\right| \geq$ MinPts. It is depicted in Fig. 3.

Density-Reachable, A point $\mathrm{p}$ is directly densityreachable from a point $p$ with respect to Eps and MinPts if there is a chain points $p_{1}, \ldots, p_{n}, p_{1}=q, p_{n}=p$ such that $p_{i+1}$ is directly-reachable from $p$. Figure 4 depicts the definition of density-reachable.

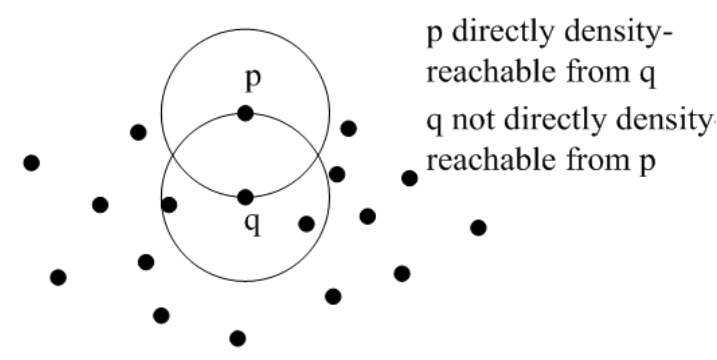

Fig. 3. Directly density-reachability

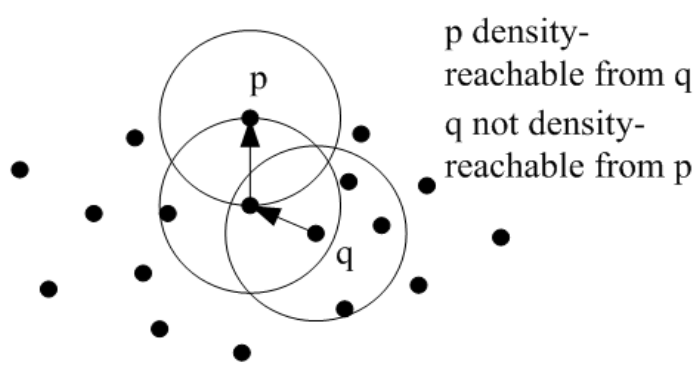

Fig. 4. Density-reachability

DBSCAN Algorithm is as follows (Ester et al., 1996):

- Define a set of data (setpoint), MinPts and Eps value

- Determine the point $p$ in the setpoint

- If the point $p$ has not yet classified, then:

- Find all points ( $p$ region) in the radius Eps and fulfill the MinPts.

- If the setpoint in the $p$ region does not fulfill MinPts, then $p$ is noise.

- If the setpoint of fulfilling MinPts $p$, then $p$ is a core point and the cluster formed.

- Repeat step 3 for all the setpoint

Figure 5 depicts the criteria of cluster in DBSCAN. Pseudocode of the basic DBSCAN algorithm is presented below (Ester et al., 1996):

DBSCAN (SetOfPoints, Eps, MinPts)

// SetOfPoints is UNCLASSIFIED

ClusterId := nextId(NOISE)

FOR I FROM 1 TO SetOfPoints.size DO

Point := SetOfPoints.get(i);

IF Point.CIId = UNCLASSIFIED THEN

IF ExpandCluster(SetOfPoints, Points, ClusterId, Eps, MinPts) THEN

END IF

ClusterId := nextId(ClusterId)

END FOR

END IF

END; // DBSCAN 


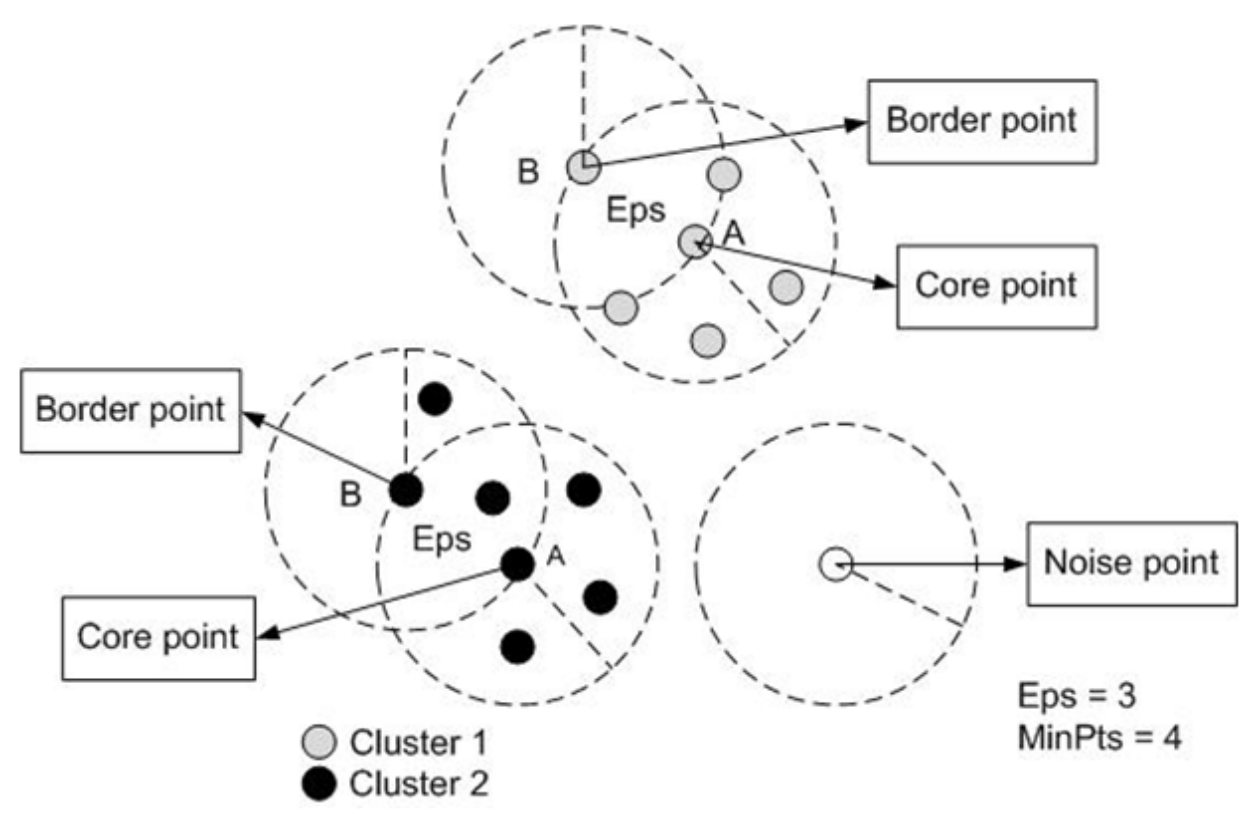

Fig. 5. DBSCAN clustering (Andrade et al., 2013)

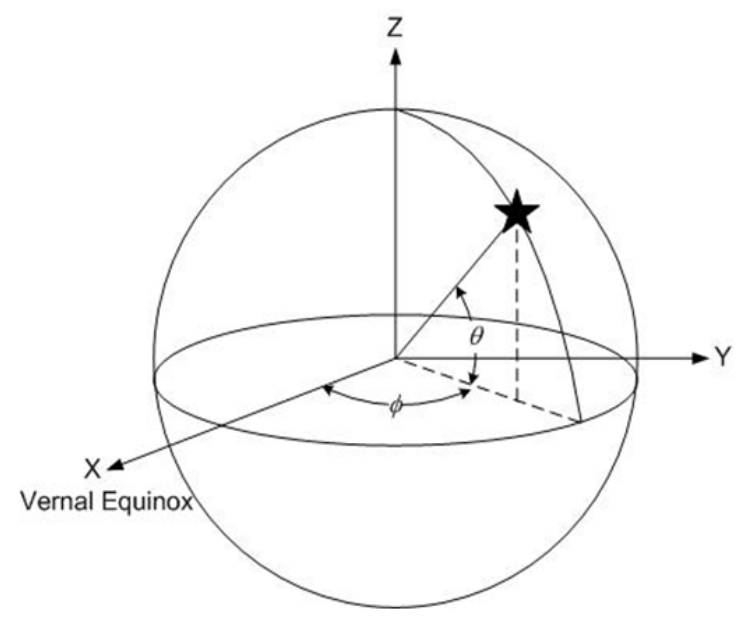

Fig. 6. Star Position in celestial coordinates

In this study, DBSCAN identify the high density region and it clusters would be created. Navigation star candidate is selected by storing only one brightest star and remove the others in a cluster. Moreover, all noises attribute also stored as a navigation star candidate. Therefore, a new star catalog consists of one brightest star in a cluster and all noises. Eps value is defined as the angle between two vectors, in this case is an angle of two stars.

Star position defines as Right Ascension (RA) and Declination (DE) in the celestial coordinate. RA is the angle of the star to the vernal equinox eastward along the celestial equator plane and DE is the angle of the star measured north or south of the celestial equator plane.
In Fig. 6, represents Right Ascension (RA) and Declination (DE) where:

$\phi=$ Right Ascension (RA)

$\theta=$ Declination (DE)

Transformation from celestial coordinate to Cartesian coordinate is as follows (Montenbruck and Gill, 2000):

$\left[\begin{array}{l}x \\ y \\ z\end{array}\right]=\left[\begin{array}{c}\cos \theta \cos \phi \\ \cos \theta \sin \phi \\ \sin \theta\end{array}\right]$

If the position of the two stars represented by the vector:

$\mathrm{a}=\left(\mathrm{x}_{1}, \mathrm{y}_{1}, \mathrm{z}_{1}\right)$
$\mathrm{b}=\left(\mathrm{x}_{2}, \mathrm{y}_{2}, \mathrm{z}_{2}\right)$

If Eps or angle between two stars is denoted by $\alpha$, so $\alpha$ is calculated by the formula:

$\cos \alpha=\frac{\mathrm{a} \cdot \mathrm{b}}{|\mathrm{a}||\mathrm{b}|}$

Since $a$ and $b$ are normalized so that $|a|=|b|=1$

So, Eps value is obtained from equation below:

$\cos \alpha=\mathrm{a} \cdot \mathrm{b}$

Thus, combine Equation 2 and 4: 


$$
\begin{aligned}
\cos \alpha & =x_{1} x_{2}+y_{1} y_{2}+z_{1} z_{2} \\
\alpha & =\cos ^{-1}\left(x_{1} x_{2}+y_{1} y_{2}+z_{1} z_{2}\right)
\end{aligned}
$$

\section{Star Catalog Generation}

The First step of DBSCAN algorithm is to determine the initial value of MintPts and Eps. The initial Eps set to a small value, e.g., 0.25 (in degree) to avoid densityreachable with high Eps value, then increased every 0.25 for next clustering loops. The clustering loops terminate if the algorithm does not create a new cluster. MinPts value set to three for each clustering process to reduce the number of stars gradually. The second step is reading star data from star catalog as setpoints. The star catalog used in the first clustering is an initial catalog contains 5103 stars. The second and subsequent clustering is using a new star catalog created from previous process.
The third step is to cluster star data using DBSCAN which is creating star clusters and star noises. Fourth step is removing all stars except the brightest one in each cluster and store the brightest star into a new catalog including all noises. Now, a new catalog is generated with less number of stars than initial catalog. Flowchart of all steps for star catalog generation is shown in Fig. 7.

DBSCAN resulted that first clustering creates 12 clusters. Figure 8 is a sample of one cluster that created in first clustering. Clustered stars are marked with red dotted while the cross marked are noises. The cluster contains about 5 stars that have ID 340, 444, 699, 2323 and 4150. Since the initial catalog list sorted by the brightness, the smallest star ID 340 is the brightest star in the cluster and it stored as a navigation star candidate including all noises as shown in Fig. 9. Result from first clustering is removed 26 stars and the number of remaining stars is 5077.

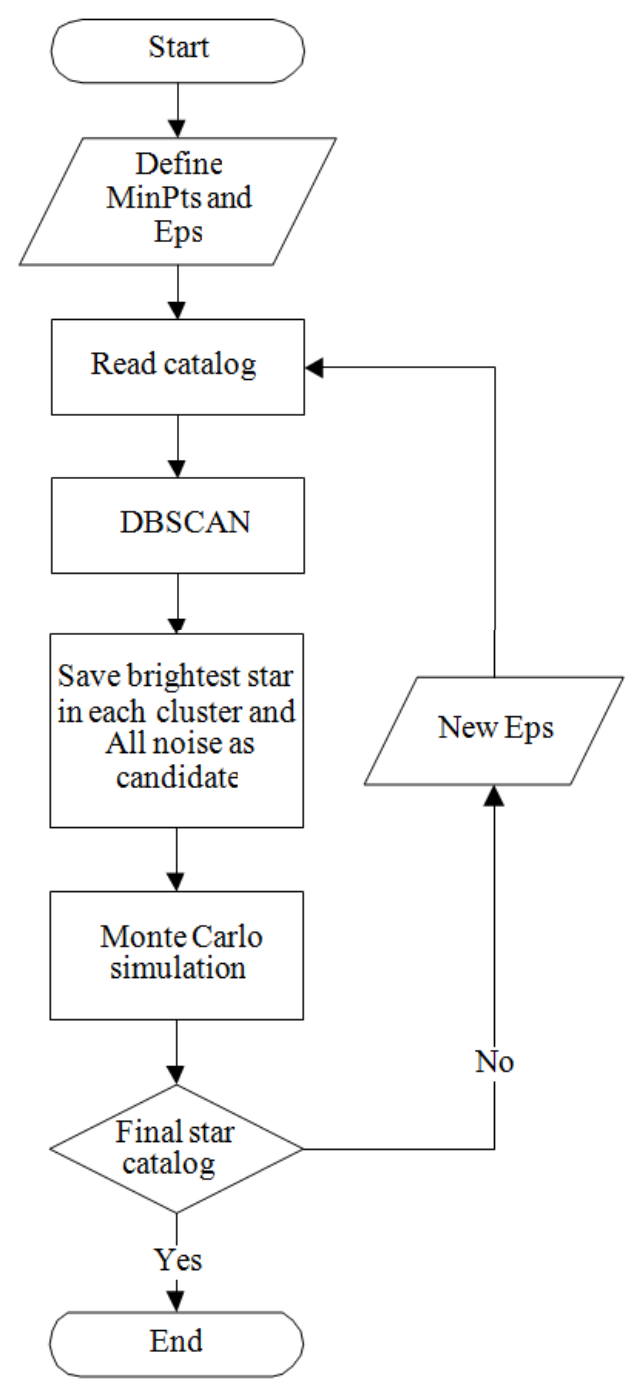

Fig. 7. Flowchart of star catalog generation 


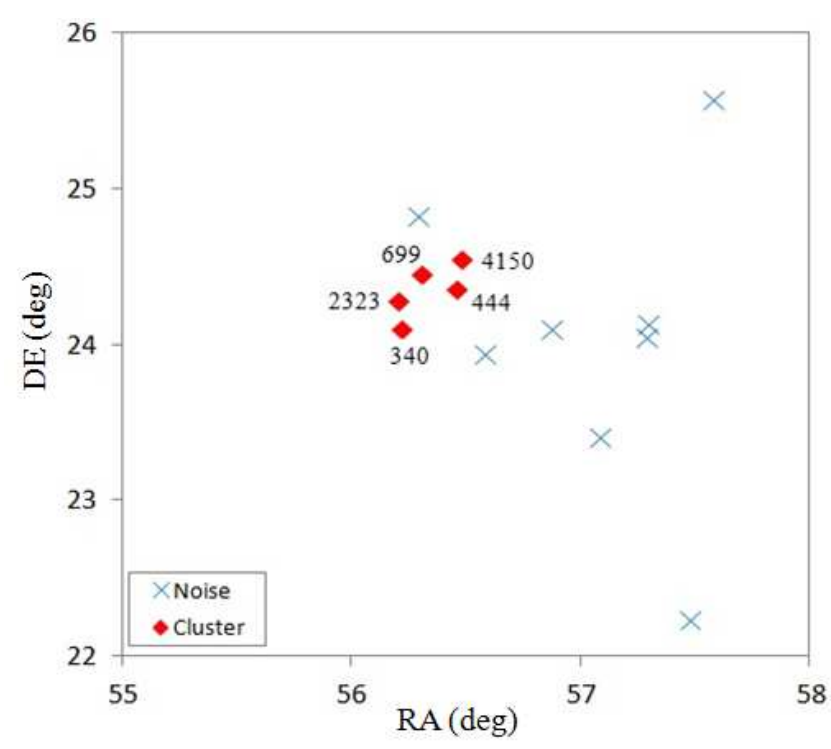

Fig. 8. A sample of one cluster created from 1st clustering with Eps $=0.25$

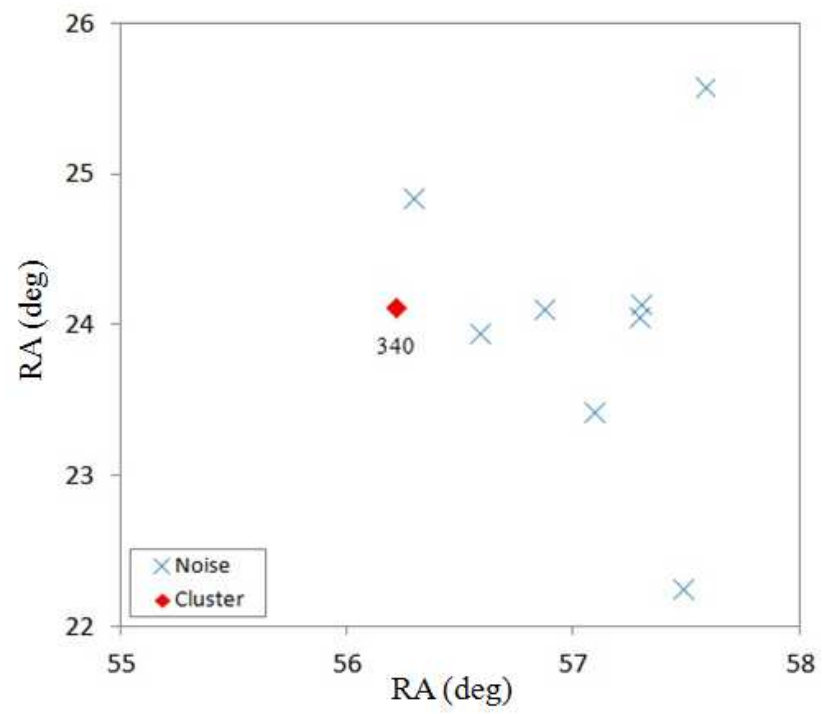

Fig. 9. Brightest star with ID 340 remains from 1st clustering

First clustering obtained number of 5077 stars that used in second clustering with Eps $=0.5$. Remaining stars from second clustering used in third clustering and so forth. The process has been performed 23 clustering and terminated while it was producing 475 stars. The consideration to terminate the clustering with number of stars 475 is it a small amount of stars to be used as navigation stars. The clustering statistics and Monte Carlo simulation result of DBSCAN and MFM is shown in Table 2.

\section{Discussion}

Monte Carlo simulation has programmed using Visual Basic 2010 Express and run using PC Intel Core i5-3470 CPU, $3.20 \mathrm{GHz}$; 4 GB RAM; Windows 7 Professional
Service Pack 1. The simulation results are shown in Table 2. It shows that for the number of stars 2555 and 1260 , obtained the percentage of success is $100 \%$ for both methods. Number of stars reduces to 846 and percentage of success is still $100 \%$ for DBSCAN but $95.7 \%$ for MFM. Next reduction numbers of stars results, lower percentage of DBSCAN also for MFM but there is a significant decreasing of percentage of success of MFM. There are interesting things that the average number of stars within FOV is almost same in the same number of stars in catalog between DBSCAN and MFM, but slightly different in a number of stars smaller than three started from the number of stars in catalog 963. It is reasonable since the distribution of stars of DBSCAN is nearly uniform than MFM as shown in Fig. 10 and 11 respectively. 
Table 2. Statistic of Monte Carlo simulation and number of star inside $23^{\circ} \mathrm{FOV}(\mathrm{S})$

Number of simulation: 1,000

\begin{tabular}{|c|c|c|c|c|c|c|}
\hline Number of stars & Method & Min & Max & Avg & $\mathrm{S}<3$ & Success $(\%)$ \\
\hline \multirow[t]{2}{*}{2555} & DBSCAN & 9 & 85 & 26.08 & 0 & 100.0 \\
\hline & MFM & 7 & 77 & 26.03 & 0 & 100.0 \\
\hline \multirow[t]{2}{*}{1260} & DBSCAN & 6 & 21 & 12.69 & 0 & 100.0 \\
\hline & MFM & 3 & 37 & 12.42 & 0 & 100.0 \\
\hline \multirow[t]{2}{*}{1084} & DBSCAN & 3 & 18 & 10.79 & 0 & 100.0 \\
\hline & MFM & 2 & 36 & 10.32 & 6 & 99.4 \\
\hline \multirow[t]{2}{*}{963} & DBSCAN & 3 & 17 & 9.49 & 0 & 100.0 \\
\hline & MFM & 1 & 31 & 9.21 & 26 & 97.4 \\
\hline \multirow[t]{2}{*}{846} & DBSCAN & 3 & 14 & 8.37 & 0 & 100.0 \\
\hline & MFM & 0 & 26 & 8.28 & 43 & 95.7 \\
\hline \multirow[t]{2}{*}{770} & DBSCAN & 2 & 14 & 7.71 & 1 & 99.9 \\
\hline & MFM & 0 & 26 & 7.67 & 66 & 93.4 \\
\hline \multirow[t]{2}{*}{681} & DBSCAN & 2 & 13 & 6.97 & 3 & 99.7 \\
\hline & MFM & 0 & 25 & 6.44 & 137 & 86.3 \\
\hline \multirow[t]{2}{*}{540} & DBSCAN & 1 & 11 & 5.21 & 34 & 96.6 \\
\hline & MFM & 0 & 18 & 5.22 & 223 & 77.7 \\
\hline \multirow[t]{2}{*}{475} & DBSCAN & 1 & 10 & 4.66 & 66 & 93.4 \\
\hline & MFM & 0 & 16 & 4.35 & 310 & 69.0 \\
\hline
\end{tabular}

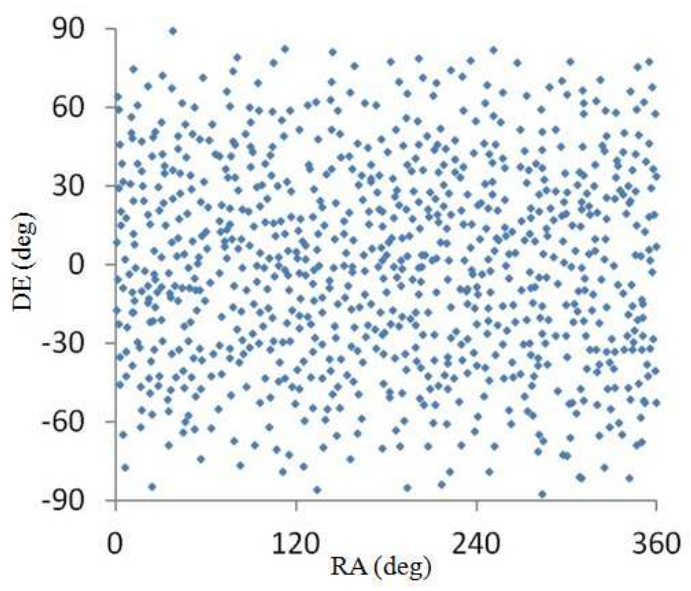

Fig. 10. Distribution of SAO catalog with 846 stars of DBSCAN

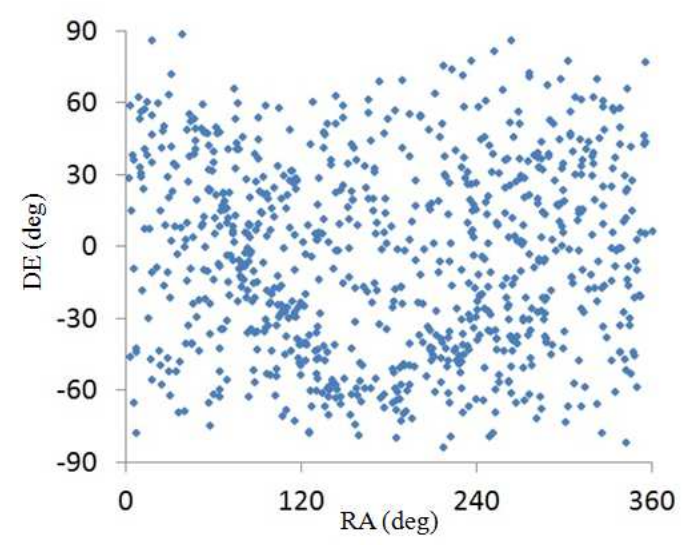

Fig. 11. Distribution of SAO catalog with 846 stars of MFM
The star density of MFM is high in Milky Way region. However, the star density by DBSCAN is near uniform celestial sphere. Comparing these figures, it has the reason that DBSCAN gives better results than MFM by Monte Carlo simulation.

\section{Conclusion}

DBSCAN is successfully applied to reduce the number of stars in order to generate star catalog. A density-based clustering method for star catalog generation is promising to obtain a star catalog reduction with the nearly uniform distribution. Monte Carlo simulation to identify minimum three stars in any FOV bore sight direction has better result for DBSCAN compare with MFM. For instance, getting number of star 846 from DBSCAN has success $100 \%$, while 846 stars from MFM is $95 \%$. The result shows that DBSCAN method is better than MFM.

\section{Future Work}

In future work, DBSCAN should be improved for star removing method in a cluster. To ensure the star distribution is more uniform, saving star by brightness is neglected. Removing star by its position in the cluster is promise likely and improves the value of Eps and MinPts.

\section{Funding Information}

The authors have no support or funding to report.

\section{Author's Contributions}

All authors equally contributed in this work. 


\section{Ethics}

This article is original and contains unpublished material. The corresponding author confirms that all of the other authors have read and approved the manuscript and no ethical issues involved.

\section{References}

Andrade, G., G. Ramos, D. Madeira, R. Sachetto and R. Ferreira et al., 2013. G-DBSCAN: A GPU accelerated algorithm for density-based clustering. Procedia Comp. Sci., 18: 369-378. DOI: $10.1016 /$ j.procs.2013.05.200

Bak, T., 1999. Spacecraft Attitude Determination-A Magnetometer Approach. PhD Thesis, Aarhus University.

Cole, C.L. and J.L. Crassidis, 2006. Fast star-pattern recognition using planar triangles. J. Guidance Control Dynam, 29: 64-71. DOI: 10.2514/1.13314

Ester, M., H.P. Kriegel, S. Jorg and X. Xu, 1996. A density-based algorithm for discovering clusters in large spatial databases with noise. Proceedings of 2nd International Conference on Knowledge Discovery and Data Mining, (KDD-96), Aug., 2-4, AAAI Press, USA, pp: 226-231.

Gai, E., K. Daly, J. Harrison and L. Lemos, 1985. StarSensor-based satellite attitude/attitude rate estimator. J. Guidance Control Dynamic, 8: 560-565. DOI: $10.2514 / 3.56393$

Kim, H.Y. and J.L. Junkins, 2002. Self-organizing guide star selection algorithm for star trackers: Thinning method. Proceedings of the IEEE Aerospace Conference, (IAC' 02), IEEE Xplore Press, USA, pp: 2275-2283.

DOI: 10.1109/AERO.2002.1035394

Kolomenkin, M., S. Pollak, I. Shimshoni and M. Lindenbaum, 2008. Geometric Voting Algorithm for Star Trackers. IEEE Tran. Aerospace Electronic Syst., 44: 441-456.

DOI: 10.1109/TAES.2008.4560198

Lindsey, C.S., T. Lindblad and A.J. Eide, 1997. Method for star identification using neural networks. Proceedings of the SPIE 3077, Applications and Science of Artificial Neural Networks III, Apirl, 4, SPIE, USA, pp: 471-478. DOI: 10.1117/12.271545
Mohammadnejad, S., A. Rostami and M.N. Sarvi, 2012. A practical view to database generation of star identification algorithm for space applications. Proceedings of the 8th International Symposium on Communication Systems, Network and Digital Signal Processing, Jul. 18-20, IEEE Xplore Press, Poznan, pp: 1-4.

DOI: 10.1109/CSNDSP.2012.6292730

Montenbruck, O. and E. Gill, 2000. Satellite Orbits: Models, Methods and Applications. 1st Edn., Springer, New York. ISBN-10: 978-3-642-63547-2, pp: 25.

Nagpal, P.B. and A.P. Mann, 2011. Comparative study of density based clustering algorithms. Int. J. Comp. Applic., 27: 44-47. DOI: 10.5120/3341-4600

Paladugu, L., B.G. Williams and M.P. Schoen, 2003. Star Pattern Recognition for Attitude Determination Using Genetic Algorithm. Proceedings of the 17th AIAA/USU Conference on Small Satellites, (CSS; 03), Aug. 11-14, Logan,

Pham, M.D., K.S. Low and S. Chen, 2013. An autonomous star recognition algorithm with optimized database. IEEE Tran. Aerospace Electronic Syst., 49: 1467-1475. DOI: 10.1109/TAES.2013.6557999

Rousseau, G.L., J. Bostel and B. Mazari, 2005. Star recognition algorithm for APS star tracker: Oriented triangles. IEEE Aerospace Electronics Magazine, 20: 27-31. DOI: 10.1109/MAES.2005.1397146

Samaan, M.A., C. Bruccoleri, D. Mortari and J.L. Junkins, 2003. Novel techniques for creating nearly uniform star catalog. Proceedings of the AAS/AIAA Astrodynamics Specialist Conference, (AAS'03), Univelt, USA, pp: 13-13.

Sohrabi, S. and A.A.B. Shirazi, 2010. A new star identification algorithm based on fuzzy line pattern matching. Proceedings of the 25th International Conference of Image and Vision Computing New Zealand, Nov. 8-9, IEEE Xplore Press, Queenstown, pp: 1-7. DOI: 10.1109/IVCNZ.2010.6148847

Zhang, C., C. Chen and X. Shen, 2004. A new guide star selection algorithm for star tracker. Proceedings of the 5th World Congress on Intelligent Control and Automation, Jun. 15-19, IEEE Explore Press, China, pp: $5445-5449$.

DOI: 10.1109/WCICA.2004.1343772 